\title{
Somatic Chromosome Structure (Observations with the light microscope)
}

\author{
G. Giménez-Martín, J. F. López-Sáez and A. González-Fernández \\ Sección de Citología, Instituto de Edafología y Biología Vegetal, \\ C. S. I. C., Madrid, Spain
}

Received June 4, 1963

The somatic chromosome structure has been studied by many cytologist. However, the proximaty of this structure to the resolving power of the light microscope has give rise to our short comprehension on it.

The existence of the sheath and of the matrix (Cleveland 1949, Kaufmann 1960, Schrader 1953, Swanson 1942, Swanson 1960), the number of chromonemata possessing the chromosomes during the different periodes of the cell division cycle remain to be explained (Kaufman 1956, Kaufmann 1960, Heitz 1931, Ris 1945, Ris 1962 and Tjio and Levan 1956). By special treatments with ammonia fumes, hot water or $\mathrm{KCN}$ has been possible to show the coiled structure of the metaphase chromosomes (Swanson 1960).

The hypothesis of the monostranded anaphase chromosomes and its duplication at the early prophase (Darlington 1937) and, on the other hand, the considerations by many other authors on double stranded or polynemic structure of the anaphase chromosome indicate our short knowledges on this matter (Darlington 1955, Ris 1961, Steffensen 1959).

\section{Materials and methods}

Root-tips of Sicilla non-scripta without or with a pretreatment 8-oxiquinoline $0.02 \mathrm{M}$ for $3-4$ hours and the staining technique of Tjio and Levan (Tjio and Levan 1950) were employed. The photomicrographies were obtained with a Photomikroskop Zeiss on microfille Kodak film.

\section{Observations}

The somatic chromosomes of $S$. non-scripta (=Endymion non-scriptus) are specially adequated for the study of the coiling structure. The 8-oxiquinoline stops the cell division at metaphase by reason of its a-mitotic action (Giménez-Martín and López-Sáez 1962), but it does not affect directly the chromosome structure.

The metaphase chromosomes show the two parallel chromatids and their centromeres clearly separated. Probably, the absence of poleward emigration is caused by a energy deficience such as it was pointed out in other precedent communication (Giménez-Martín and López-Sáez 1962). 
Our observations on the chromosome coiling are as follows:

a) The prophase chromosomes present both chromatids relationally coiled about each other, i.e., the chromatids form a plectonemic coil. Each chromatid is made up by two clearly visible subchromatids. Moreover, these two



Fig. 1. Metaphase chromosomes. Every chromosome is formed up by two chromatids and every chromatid by two subchromatids. Some chromatids are plectonemically coiled and each chromatid is made up by two subchromatids. These subchromatids coiling about each other form other plectonemic coil within the chromatid.

subchromatids, coiling about each other, form other plectonemic coil within of the chromatid (Fig. 1a). 
b) In the metaphase chromosomes the gyre number is smaller than in the prophase chromosomes, the chromatid length decreases and, at the same time, the chromatid increases in diameter. Thus, the decrease in gyre number is accompanied by an increase in gyre diameter.



Fig. 2. Each chromatid is made up by two clearly visible subchromatid. a, c, d, the sister chromatids show different gyre situation and gyre number. b, two homologue chromosomes. Each chromatid as different spiralization.

In late metaphase the chromatids either have eliminated at all their plectonemic coil or remain a few gyres which are lost in the anaphase as 
relational coils.

c) Therefore, every metaphase chromosome is made up by two chromatids and every chromatid by two subchromatids. Every anaphase chromosome is formed up by two chromatids (subchromatids) and every chromatid (subchromatid) by two subchromatids (half-subchromatids). The chromosomes show neither matrix nor sheath.

An increase in diameter of the metaphase chromosomes arise of an increase in diameter of the chromatids. The coiled structure of each chromatid has decreased in gyre number and increased in gyre diameter of its subchromatids (Fig. 2a).

d) The chromatids of homologue chromosomes, as well as the sister chromatids of each chromosomes, show different gyre situation and gyre number; although the gyre diameter and the chromatid diameter are similar in all chromosomes of the dotation.

\section{Discussion}

1. Structure. According to Swanson (1960): “The chromonemata are supposed by most cytologist to lie embedded in a mass of achromatic material called the matrix which is bounded at its outer limits by a sheath or pellicle". However, a such structure has been vigorously denied by Darlington (1937) and Ris (1945). In the diagram of the chromosome morphology indicated by Schrader (1953), the chromosome is bounded by this sheath and the matrix is the main mass of the chromosome and that part that characteristically is Feulgen positive. It can be removed by enzymatic or chemical means, leaving a Feulgen negative strand which has been termed the residual chromosome.

a) In our plates is impossible to observe either the sheath or the matrix. The chromosomes are only made up by eight strands, associating two-by-two to form plectonemic coils in three levels of structure (Fig. 3a).

b) The chromosomes show up as a doubled structure. In a diploid individual every chromosome is represented two times, every chromosome is formed by two chromatids, all chromatids are made up by two subchromatids, and every subchromatid by two half-subchromatids (Giménez-Martin et al. 1963, Steffensen 1959). The duplication and the separation of the chromatids are facilitated by the lack of the sheath or the interchromatidic materials. The microscopical pattern of chromosome structure arises probably of a molecular coil.

2. Despiralization. By special pretreatments it is possible to show that each chromatid of a metaphase chromosome is a coiled structure. The individual gyres make up the somatic or standard coil. The turnings of one chromatid about another are termed relational coils. Actually it is considered that chromosome present a process of spiralization and other of despiralization during each cell division cycle. 


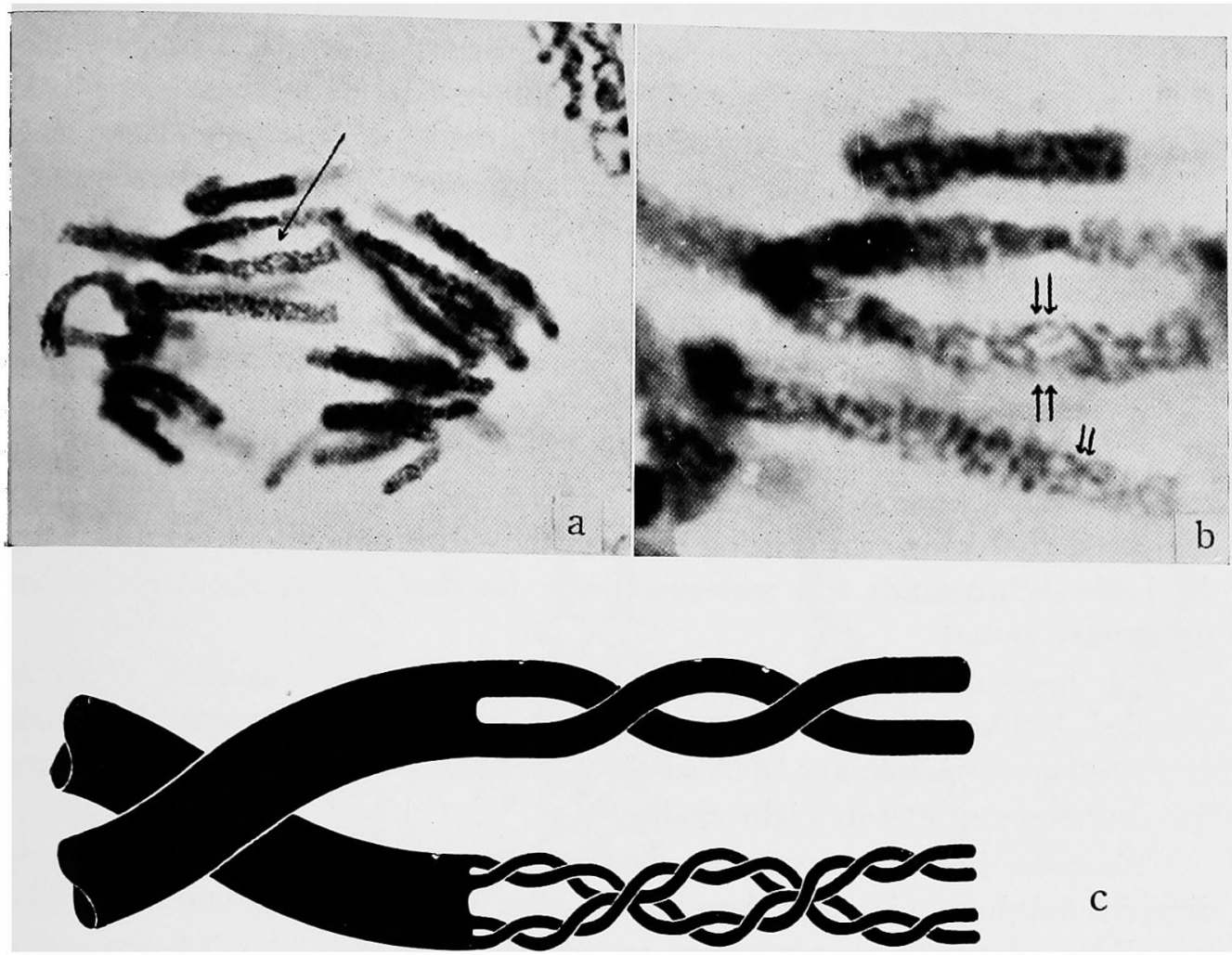

Fig. 3. The somatic structure is observed as a series of plectonemic coiling systems overlapping about each other into three different levels, chromatids, subchromatids and halfsubchromatids.

a) During cell division a continous process of despiralization is given rise. As a chromatid about other as one subchromatid about another and in agreement with this it is logical to assume that also the sub-units of each subchromatid undergo a similar despiralization.

The chromatid despiralization facilitates the poleward movement at the anaphase. Each chromatid show two subchromatids plectonemically coiled but such gyre number that their plectonemic coil can be eliminated for the next following division.

b) The continous process of despiralization gives rise to the separation of more and more single strands, but during mitotic interphase the strand number is duplicated.

c) The mechanism of despiralization has no genetic ground in its behavior, since this process has not identical development either in homologues chromosomes or in sister chromatid within chromosome.

We mean that process has only a mechanic character being regulated indirectly by the genetic information.

d) During division cycle the chromosomes undergo a process of con- 
traction from prophase to anaphase, and a process of enlargement from anaphas to interphase. The chromosome contraction arises by increase in gyre dii meter within subchromatids and the gyre number decreases at the same tim It is logical to assume that the elemental strands do not modificate the length, but that chromosome contraction arises only by the above mentione mechanisms. Secondary, when the chromosomes shorten, the chromatic disengage themselves and come to lie side by side, the chromatids as preparated for the poleward emigration.

e) We pointed out that the denominations of somatic or standard coi and relic coils have a little significance since, according with our hypothesi: all coilings are loose spirals, giving rise from a molecular helix. Hence w mean as more appropriated to term $\alpha$ helix that existing about each othe chromatid: $\beta$ helix that existing between the subchromatids; $\gamma$ helix betwee the half-subchromatids and molecular helix for that coiling about each oth elementary strand.

3. Replication. Since the Watson-Crick's model assumes a doub: stranded DNA molecule, we conclude that individual subchromatids contai at least two complementary DNA chains, chromatids at least four chains an the chromosome at least eight chains.

Since we have observed that the chromatid is formed by two clearl different subchromatids and therefore suggest that one chromatid has at leas four DNA chains it is appropriate to review the models of DNA replicatio suggested by Taylor (Taylor 1958, Taylor 1958, Taylor 1959, Woods 1959 As a result of the experiments with tritiated thymidine Taylor concluded the each DNA chain synthetizes during interphase, a complementary copy, unitin the template with its copy to form the chromatid of the subsequent ce

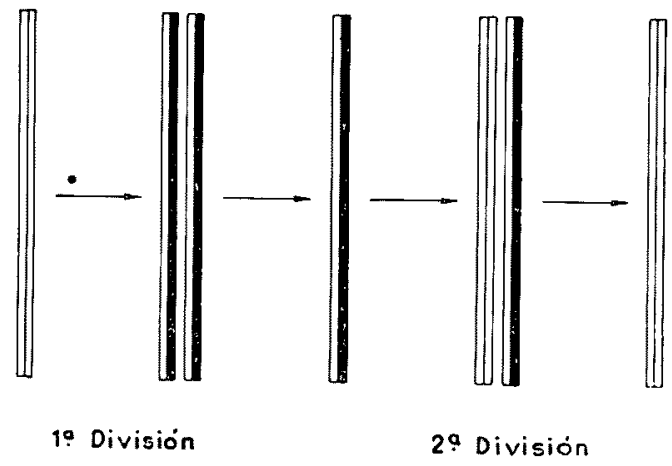

Diagram 1 division. This can be de monstrated diagrammat cally as Diagram 1.

If we accept Taylor hypothesis for DNA rt plication but introduce or suggestion, that is the each chromatid has at lea: four DNA chains on would anticipate the follow ing outcome (Diagram 2

\section{Note}

- In the diagram 2 and 3 each two complementary chains were outlined in parale form a better understanding of the picture.

- Tritiated thymidine treatment. 
Since the outcome (Diagram 2) is clearly at variance with Taylor's observations (Diagram 1) we would like to propose that Tailor's hypothesis needs modification so as to explain how the newly synthesized DNA chains can remain together or he reunited following replication. They can be schematically shown in Diagram 3.

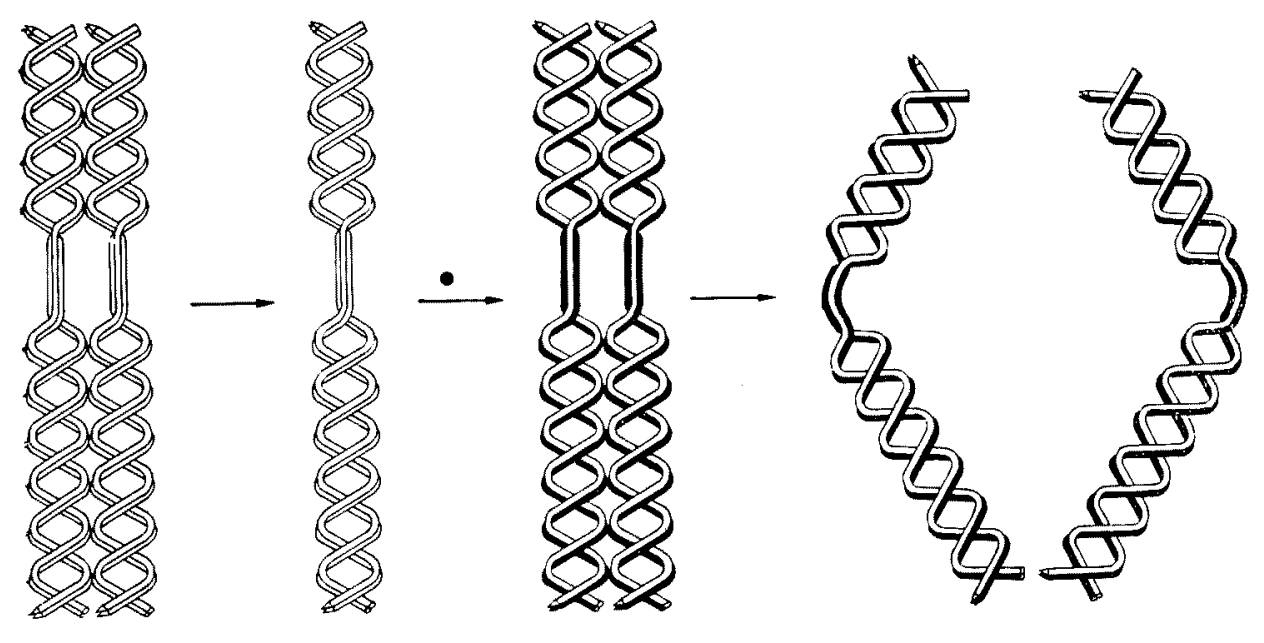

19 Division
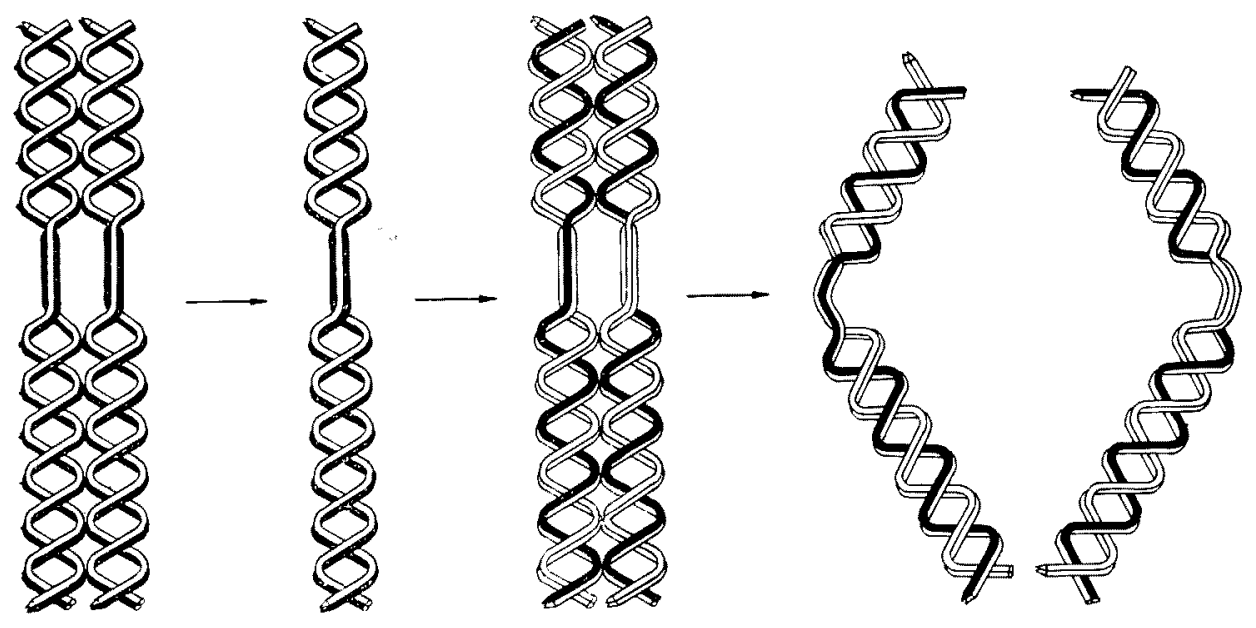

\section{2: Division}

Diagram 2

It is noteworthy to point out this hypothesis explains Tailor's experiments even if the number of DNA chains in the chromosome is higher than eight. 
As a result of this last diagram we came to the conclusion the physiological unit of the chromosome is not the chromatid that the half chromatid independent of its stands number.
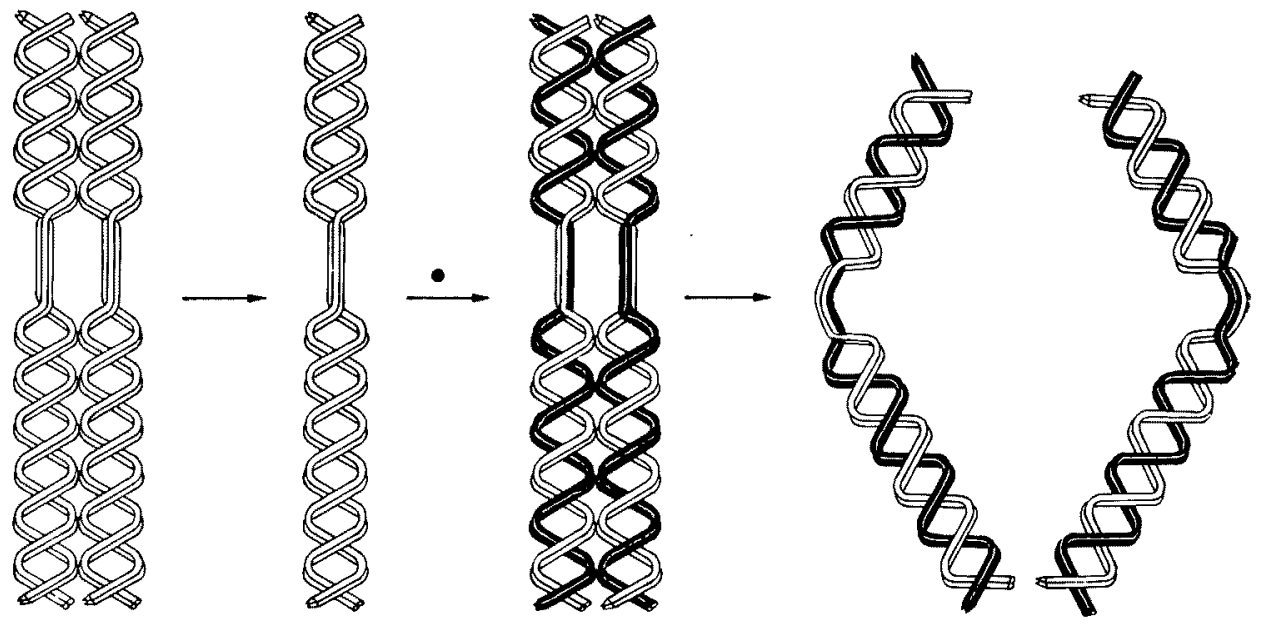

19 Division
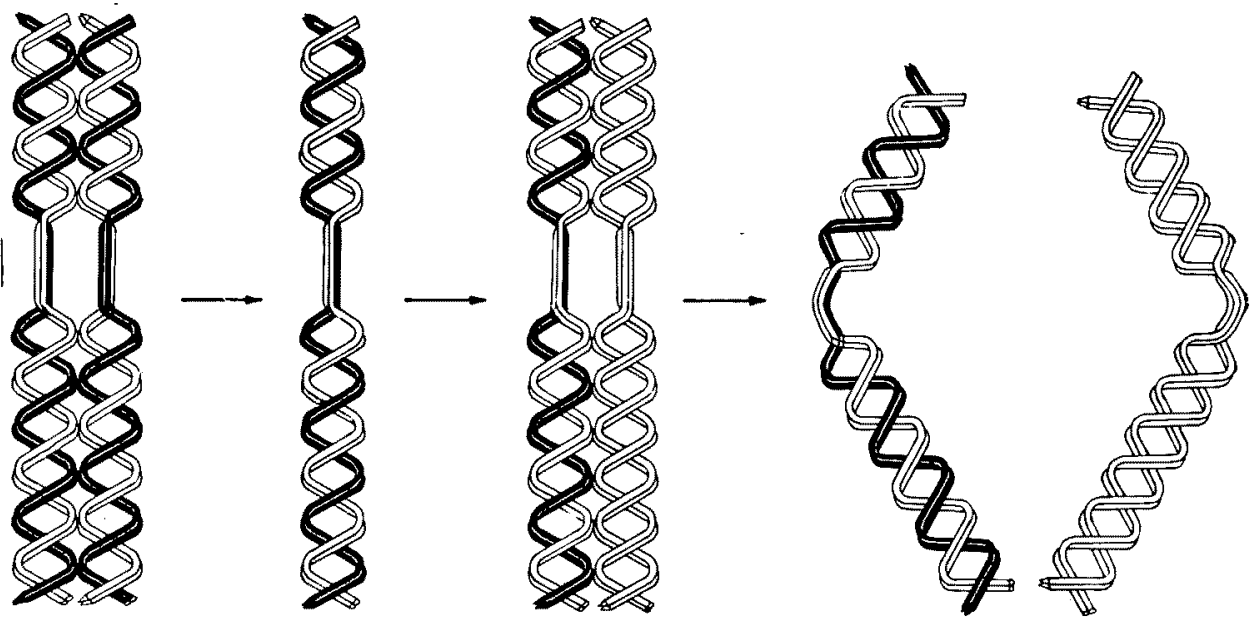

2: Division

Diagram 3

\section{References}

Cleveland, L. R. 1949. Trans. Am. Phil. Soc. 39: Pt. 1.

Darlington, C. D. 1937. Recent advances in Cytology. 2, Edition Blakistsn, Phil.: 671.

- 1955. Nature 176: 1139.

Giménez-Martín, G. and López-Sáez, J. F. 1962. Fiton 18 (1): 15.

-, - and González-Fernández, A. (Submitted to Experientia). 
Heitz, E. 1931. Planta 12: 775.

Kaufmann, B. P. 1948. Botan. Rev. 14: 57.

- and De, D. N. 1956. J. Biophys. Biochem. Cytol. 2. Supl. 419.

—, Gay Helen, and McDonald, M. R. 1960. Internat. Rev. of Cytol. XI.

Ris, H. 1945. Biol. Bull. 89: 242.

- 1961. Canadian Journal of Genetics and Cytology 2 (2): 95.

Schrader, F. 1953. Mitosis. 2nd Ed. Columbia University.

Steffensen, D. 1959. Brookhaven Symposia in Biology, n. 12: 103.

Swanson, C. P. 1942. Meiotic coiling in Tradescantia. Bot. Gaz. 10s. 457.

- 1960. Cytologie und Cytogenetik.

Taylor, J. H. 1958. The organization and duplication of genetic material. Proc. X Int. Cong. Genetics Vol. 1.

- 1958. Scientific American V. 198: 36.

- 1959. A symposium on molecular biology. Chapter 21: 304.

Tjio, J. H. and Levan, A. 1950. An. Aula Dei 2 (1): 21.

- and - 1956. An Aula Dei, 4. 185.

Watson and Crick, F. H. C. 1953. Nature 171: 964.

Woods, P. S. and Schairer, M. U. 1959. Nature 183: 303. 